\title{
Elementos necesarios para una modalidad de educación a distan- cia en bibliotecología
}

\author{
Brenda Cabral Vargas *
}

Artículo recibido:

14 de noviembre de 2007.

Artículo aceptado:

18 de junio de 2008.

\section{RESUMEN}

Se hace referencia a los principales elementos que deben considerarse al planear una modalidad a distancia en bibliotecología. Estos elementos son el resultado del análisis realizado a diferentes fuentes, tanto nacionales como internacionales, y de la información obtenida a partir de tres programas de estudio en bibliotecología en América Latina. La planeación se sugiere por etapas, lo cual permitirá que cualquier institución interesada en implementar una modalidad a distancia en esta disciplina cuente con un panorama general sobre lo que tiene que considerar al planearla y ponerla en marcha. No se pretende aquí manejar o definir conceptos de forma exhaustiva, sino establecer la relación entre ellos.

* Centro Universitario de Investigaciones Bibliotecológicas de la UNAM, México. brenda@cuib.unam.mx

INVESTIGACIÓN BIBLIOTECOLÓGICA, Vol. 22, Núm. 46, septiembre/diciembre, 2008, México, ISSN: 0187-358X. pp. 59-89 
Palabras claves: Educación a distancia; Proceso de enseñanza-aprendizaje; Elementos de la educación a distancia en bibliotecología.

\begin{abstract}
Necessary elements for distant education in Library Science Brenda Cabral Vargas

Reference is made to the main elements which should be considered in planning a distant education mode in Library Science. These are the result of an analysis made on different national and international sources plus the information obtained from three Latin American curricula. The planning proposed would allow any interested institution wanting to implement this mode a general view on what should be considered to set it in motion. However, there is no pretension of exhaustive defining or handling of concepts.
\end{abstract}

Keywords: Library Science; Distant education; Teaching-learning process.

\title{
INTRODUCCIÓN
}

T a Sociedad actual ha impuesto un ritmo acelerado de cambios en todos Llos niveles y dentro de este marco de dinamismo la educación ha adquirido un valor muy especial, debido a que el conocimiento se ha convertido en un factor estratégico para el desarrollo social, político, económico y cultural del individuo.

México está inmerso en un proceso de cambio tanto nacional como regional e internacional en que ha influido a sus diferentes sectores educativos.

Considerando que nuestro país está conformado por una población eminentemente joven que cada año demanda servicios de formación profesional en el ámbito superior, y que actualmente la educación superior cubre sólo un porcentaje de la demanda, dependiendo del Estado del que se trate, debemos de considerar a la educación a distancia como una opción para satisfacer esta necesidad educativa.

Así la finalidad de este artículo es identificar, seleccionar y proponer los mecanismos, parámetros, criterios y requisitos mínimos que debe tener una 
modalidad de educación a distancia en bibliotecología. Para ello nos basaremos en la teoría general de sistemas, ya que

La construcción de modelos en la mayoría de los países latinoamericanos se encuentra en un proceso de construcción, comenzándose a advertir los primeros resultados a partir de los estándares desarrollados por cada país. ${ }^{1}$

Esta teoría permitirá entender cuáles son los elementos que se requieren, así como la manera en que se da la interacción holística entre éstos, para permitir el funcionamiento adecuado de una modalidad a distancia en bibliotecología.

Para lograr lo anterior, partimos tanto de bibliografía nacional como internacional, y del análisis de modelos educativos a distancia en bibliotecología en otros países de América Latina. Intentamos detectar y seleccionar los elementos que han favorecido el éxito de aquellos programas que han podido desarrollar con calidad las competencias profesionales pertinentes en los futuros profesionales de la bibliotecología y estudios de la información.

\section{ELEMENTOS QUE INTEGRAN UNA MODALIDAD A DISTANCIA}

La modalidad a distancia en bibliotecología está integrada por varios elementos que la caracterizan: los materiales de aprendizaje, los asesores, los objetos y espacios de aprendizaje. Pero hay que decir que una modalidad de este tipo la integran mucho más elementos que permiten el adecuado funcionamiento de ésta.

Pero ¿por qué investigar cuáles son los elementos de una modalidad de educación a distancia en bibliotecología? La respuesta es que hoy existe una acelerada generación y renovación de conocimientos científicos y tecnológicos, lo que hace que muchos de los conocimientos resulten obsoletos a corto plazo, y esto hace que la modalidad educativa más idónea para dar respuesta inmediata a las necesidades de formación y actualización de profesionales sea la "educación a distancia". En nuestra disciplina el requerimiento de profesionales de la información ha tenido siempre una gran demanda, pero debido a que muchos de ellos no tienen acceso a instituciones educativas que los formen en su área de residencia, no se pueden dedicar a esta actividad de manera profesional. Por ello, y para no tener que trasladarse físicamente de su

1 Encuentro de directores y encuentro de docentes de escuelas de bibliotecología y ciencias de la información ( $7^{\circ}$ y $6^{\circ}$ : 2004: Mar de la Plata, Argentina), Argentina, MERCOSUR, 2005. 
lugar de origen, han recurrido a esta modalidad. Gracias a que estos participantes (estudiantes y personal docente) pueden ubicarse en cualquier punto del espacio territorial, y a que sus métodos y recursos técnicos permiten una rápida actualización y difusión de conocimientos, esta modalidad está siendo valorada cada día más por las instituciones de educación superior de México, así como por las autoridades educativas de nuestro país en general.

También sucede en muchas ocasiones, que la situación personal de los profesionales les impide asistir a programas presenciales de formación y/o actualización, y en estos casos la educación a distancia resulta una opción idónea para poder participar en dichos programas sin las limitaciones que implican los horarios fijos, los traslados físicos e incluso los cambios temporales de residencia.

Las modalidades a distancia en las instituciones educativas que forman profesionales en bibliotecología pueden contribuir a ampliar la oferta educativa y el desarrollo de nuevos programas en los diferentes niveles educativos. También contribuirán a ampliar la oferta de educación continua.

Por ende esta modalidad educativa tendrá estrecha relación con el sistema de redes académicas y hará posible una gran movilidad, porque acrecentará la posibilidad de que existan programas de estudio de tipo interinstitucional que permitirán reunir a docentes y alumnos que se encuentran dispersos en el extenso territorio nacional; y nos ayudará a aprovechar el potencial de los docentes que se encuentran fuera del país.

La educación a distancia en bibliotecología, como en cualquier otra área del conocimiento, exige una madurez psico-social que les permita a las personas asumir responsablemente el compromiso de trabajar y aprender por sí mismos, voluntariamente. En este tipo de aprendizaje es el propio estudiante quien organiza su tiempo, su esfuerzo y hace su currículum, ya que los programas de educación a distancia contiene una mayor variedad, aunque la misma calidad que los programas de otras institucioness que no practican esta modalidad.

En la modalidad de educación a distancia es muy importante tener disciplina y hábitos de estudio independientes, pues como no se está dentro de un ambiente escolar es muy fácil que las personas puedan perder la motivación por seguir estudiando.

Para que una modalidad de educación a distancia funcione adecuadamente deben estar presentes varios elementos, dependiendo siempre del nivel de desarrollo al que se apunte. Para fines de este artículo hemos considerado tres etapas: planeación, implementación y consolidación. A continuación se muestra un cuadro que incluye todos los elementos que integran un modelo de educación a distancia, así como la interacción de estos elementos: 


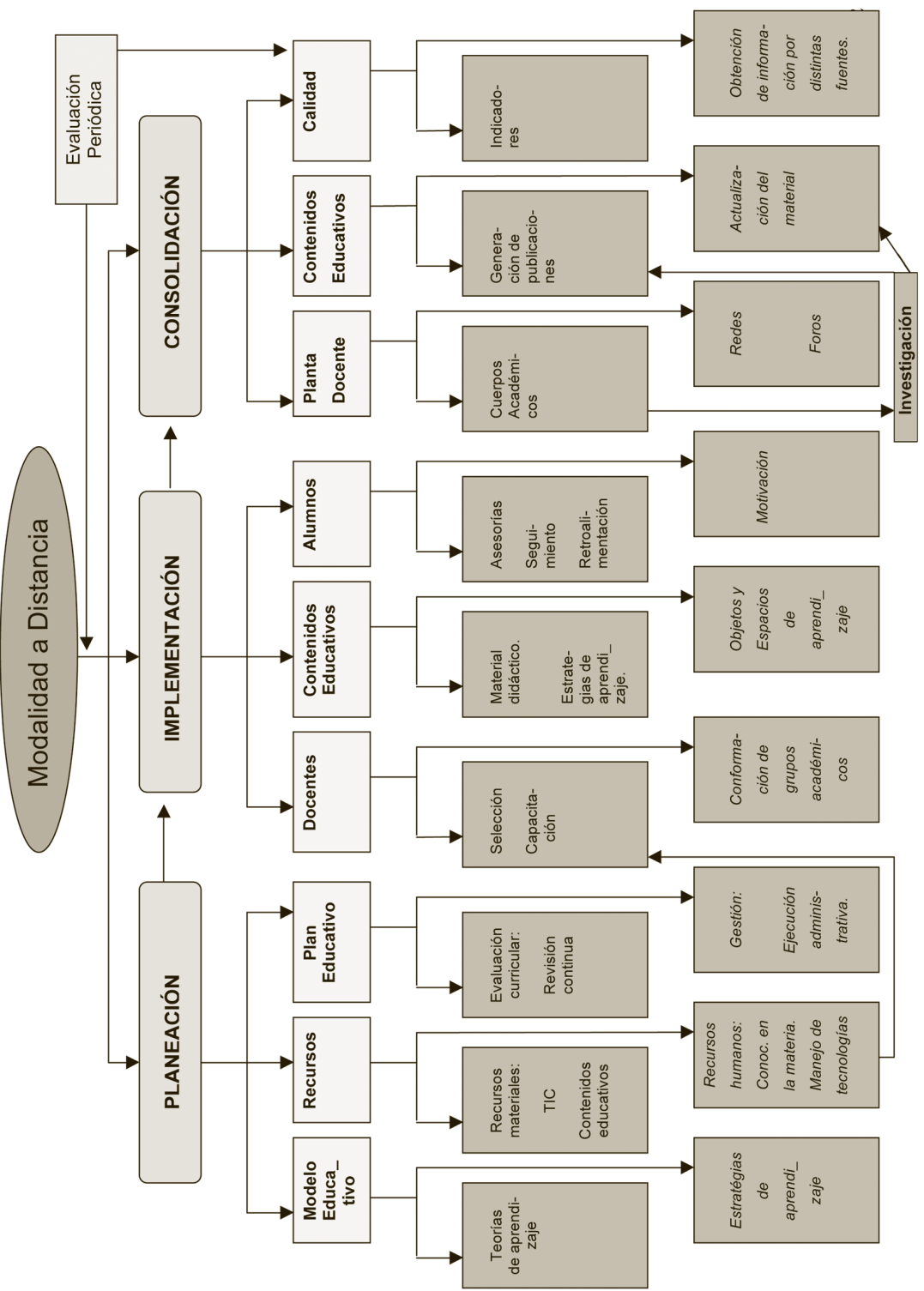


En esta primera etapa se deben considerar todos los aspectos que integran un proyecto, como son los objetivos, los propósitos, el perfil del estudiante y del egresado, los currículos, los contenidos, los docentes que estarán a cargo de dicho programa y todo lo que se va a requerir para el funcionamiento de dicha modalidad. ${ }^{2}$ La planeación propondrá metas que deberán alcanzarse en determinados lapsos, con la finalidad de ir marcando prioridades. Además en esta etapa no se pueden pasar por alto las teorías de aprendizaje, que serán el eje en torno al cual girará toda la planeación, y éstas estarán enmarcadas dentro del modelo educativo que se seleccione. Un modelo, sostiene Garduño,

[...]se refiere a una amplia gama de tipologías y aplicaciones, y las particularidades que éstas pueden adoptar dependen de la disciplina de la que se derivan. En consecuencia, la noción de modelo puede hacer referencia desde un conjunto de ideas abstractas hasta cualquier representación tangible. ${ }^{3}$

Si bien plantear un modelo académico implica conocer previamente la misión y la visión de la institución educativa, así como una serie de referentes que se muestran en el marco conceptual, también incluye el hecho de que cada elemento sea clave para garantizar la calidad de la educación. Claro que se debe tener un eje principal y que todos los procesos no pueden girar alrededor de él, más bien los que debe garantizarse es que exista un equilibrio entre los tres ejes principales del proceso enseñanza-aprendizaje.

En función de lo expuesto por varios estudiosos de la pedagogía como Colom $^{4}$, Bustamante 5 y otros expertos en la materia, consideramos que el modelo pedagógico es aquello que sustenta al modelo educativo en tanto que las distintas teorías de aprendizaje estructurán las estrategias de aprendizaje, los objetivos, el currículo y, en general todos los elementos que van a interactuar en el proceso enseñanza-aprendizaje basado en esta modalidad.

2 Se podrán tomar algunos ejes como referencia, por ejemplo: modelo educativo, aprendizaje, tecnologías, materiales, administración y algún otro que sea importante para la institución.

3 Roberto Garduño Vera. Modelo para la enseñanza vía internet de una especialización en organización de recursos informativos digitales, Madrid, el autor, 2002, p.177. Tesis (Doctorado en ciencias de la información) Universidad Complutense de Madrid. Facultad de Ciencias de la Información, Departamento de Biblioteconomía y Documentación.

4 A. J. Colom Canellas. Pedagogía tecnológica para la educación a distancia. En los nuevos escenarios y las transformaciones tecnológicas, p.30.

5 H. G. Bustamante Rojas. Propuesta de un modelo de orientación educativa a distancia; En Educación y formación a distancia. Práctica, propuestas y reflexiones. México, Universidad de Guadalajara, 2001, p.172. 


\section{Teorias de aprendizaje}

El concepto de aprendizaje que se manejará dentro del trabajo será el de aprendizaje independiente, el cual, como cree Piaget,

[...] es el resultado de una construcción de estructuras intelectuales con materiales tomados de la cultura circundante, a partir de actividades que tienen sentido y significado para el individuo, ${ }^{6}$

Este aprendizaje estará apoyado por un conjunto rico en materiales y objetos de aprendizaje y también por la creación de espacios virtuales adecuados para generar conocimientos en la bibliotecología. Lo anterior es lo más adecuado para lograr un aprendizaje significativo que permita presentarlo como un proceso continuo, enriquecedor, progresivo e integrador que ayude al estudiante a adoptar y generar los conocimientos, destrezas y actitudes requeridas en su formación.

Durante la investigación y desarrollo de las ideas planteadas en este artículo se nota que la flexibilidad, la equidad, la eficacia en costos, la cobertura, la descentralización y la alternativa educativa son algunas de las cualidades que deben ser consideradas en cualquier modalidad a distancia, lo cual, sin embargo, para que quede entendido y mejor aplicado en cualquier currículo tendrá que analizar primeramente el o los modelos que quieren ser utilizados en una institución determinada. De este modo se comprenderá cómo y en que forma interactúan los diferentes elementos que se relacionan en éste tipo de modalidad de enseñanza-aprendizaje, y que se llevan a cabo en este tipo de modalidad educativa, pero deberá considerarse en todo momento la teoría de sistemas en esta investigación y planeación y observarse cómo influyen cada uno de los supuestos teóricos, en especial los metodológicos, que propician la real asimilación de conocimiento por parte del estudiante. Las herramientas tecnológicas son consideradas como el vehículo para llevar a cabo dicho aprendizaje, ya que no se pueden quedar fuera de análisis y evaluación las diferentes estrategias que se utilizan en nuestra disciplina para facilitar el acceso a los contenidos, así como los roles que actualmente cumplen los estudiantes, docentes y los materiales instruccionales, lo cual no sólo tendrá un punto de vista único, sino que debe ser evaluado y analizado desde el punto de vista académico y tecnológico.

Para poder describir cabalmente cómo se da el proceso de enseñanzaaprendizaje en la modalidad a distancia hubimos de leer varios autores, que

6 Jean Piaget. Seis estudios de psicología. p.67. 
se citan en el cuadro uno. Dichos autores tienen varias concepciones (que también se describen en dicho cuadro), que permiten ver este proceso desde distintas aristas. Por ejemplo, se consideraron los aportes sobre la teoría de la comunicación representada en las apreciaciones de

Jesús Martín Barbero, su aplicabilidad a la educación por Daniel Prieto Castillo y Francisco Gutiérrez Pérez, y necesariamente la postura desde el interaccionismo social representado por el psicólogo y lingüista soviético Lev Vigotsky, ${ }^{7}$

para quien la conducta de los seres humanos está mediada social y culturalmente.

Una de las teorías que mayor incidencia han tenido dentro de la denominada tecnología educativa es, por supuesto, el conductismo. De hecho esta teoría cobro renovada vigencia con el diseño de máquinas de enseñanza y el aprendizaje por computadora. Como es sabido, el conductivismo en su forma más simple sostiene que el aprendizaje se realiza a través del impacto que tiene en el sujeto el ambiente exterior, donde el individuo responde favorable o desfavorablemente conforme el premio o castigo que recibe por su conducta, el neoconductismo de Skinner ${ }^{8}$ es parte de esto.

Desde su aparición como teoría de aprendizaje el conductismo, presentó límites claros y se gano fuertes críticas, pues al estar dirigido a los aspectos conductuales exteriores del individuo, poca cuenta pudo dar de los procesos que vive el sujeto para llevar a cabo el aprendizaje.

Sin embargo, en este plano superficial, el conductismo ha sido retomado en nuestra disciplina para fomentar la habilidad en el manejo y la manipulación de las máquinas (lectores de microfichas, cintas magnéticas) y muy en particular de la computadora; es decir, ha sido orientado a destacar el plano elemental de habilidades básicas. La memoria por rutina y el aprendizaje de ciertas habilidades motrices son los aspectos que dicha teoría promueve en cuanto a tipos de aprendizaje.

Partimos del hecho de que esta teoría es incapaz de explicar gran parte de la actividad intelectual y que no contribuye al desarrollo del pensamiento abstracto y reflexivo. De esta suerte, aun cuando se ha incorporado parte del discurso conductista a la educación a distancia, sobre todo en cuanto al desarrollo de las habilidades básicas para el manejo de los procesadores y el software, las teorías que mayor relevancia han adquirido, cuando menos a nivel de discurso en la enseñanza de la bibliotecología y ciencias de la información, son el cognoscitivismo y el constructivismo. 
Hernández Rojas ha señalado que la teoría cognoscitivista es una de las que cobran mayor relevancia en el terreno del procesamiento de la información y el trabajo en la modalidad de educación a distancia. Entre las primeras proyecciones de aplicación en el terreno de la educación basada en las teorías de aprendizaje cognoscitivista se encuentran los trabajos de Bruner y Ausubel. El primero propuso el "aprendizaje por descubrimiento" y el "currículo para pensar", mientras que Ausubel propuso la teoría del aprendizaje significativo o de la asimilación, manifestando gran inquietud por el análisis multidisciplinario y el estudio de cuestiones educativas en contextos escolares. De estas propuestas surgió "[...] la psicología instruccional erigida sobre las ideas de Dewey, Ausubel y Glaser". ${ }^{9}$ Postura que por otro lado parte de la concepción de la educación como un proceso socio-cultural que se transmite de generación en generación y que se expresa en distintos currículos, los cuales, para ser asimilados por los estudiantes deben ser presentados y estructurados con un sentido y valor fundamental para que los estudiantes aprendan de manera significativa; también deben ser planificados y organizados para crear las condiciones necesarias mínimas, además de generar un contexto propicio que haga intervenir al alumno activamente en su dimensión cognitiva (conocimientos previos) y motivacional-afectiva (disposición para aprender y creación de expectativas que despierten la creatividad). El estudiante por su parte, deberá desarrollar habilidades intelectuales y estratégicas adecuadas para conducirse ante cualquier tipo de situaciones de aprendizaje, así como para aplicar los conocimientos adquiridos frente a situaciones nuevas.

Por otra parte, la teoría que ha apoyado el discurso de la educación a distancia es la que Bates denomina Psicología bumanista, la cual se resume en lo siguiente:

Cada individuo existe en un mundo continuamente cambiante de experiencias donde él es el centro -centro desde el cual interpreta el mundo exterior de ahí que el conocimiento- sea construido por cada individuo mediante la interpretación y comprobación del significado de sucesos externos en términos de la relevancia de la experiencia pasada de cada individuo. ${ }^{10}$

En este terreno el docente centra su función en la planeación y organización de experiencias didácticas que no se restringen a la transmisión de información sino que se proponen la promoción, la inducción y la enseñanza de habilidades o estrategias cognitivas que les permitan a los alumnos explorar,

9 Gerardo Hernández R "Proyecciones de aplicación del paradigma cognitivo al contexto educativo”, En Paradigma en psicología para la educación. Piados-educador, 1997, p.97.

10 T Bates. Op. Cit. p.58. 
experimentar, solucionar problemas y reflexionar sobre temas definidos de antemano, y otras tareas diversas o actividades que surjan de la inquietud de los propios alumnos y les proporcionen apoyo y retroalimentación continuas ayudando a crear un clima propicio para que el alumno experimente autonomía y competencia que les permitan atribuirles valor a sus propios logros y esfuerzos.

Parte importante de lo que destaca esta teoría es que

[...] en cualquier contexto escolar, por más restrictivo que éste sea, siempre existe en el alumno cierto nivel de actividad cognitiva, éste nunca es un ente pasivo a merced de las contingencias ambientales o instruccionales[...] En consecuencia, el diseño instruccional, en cualquier ámbito educativo, exige partir de lo que los alumnos ya saben[...] y, con base en ello, programar las experiencias sustanciales dirigidas a promover nuevos aprendizajes con sentido para los mismos. ${ }^{11}$

Si bien es cierto que estas teorías en ocasiones se utilizan de manera aislada, es recomendable que se combinen entre sí para hacer más enriquecedor el proceso de enseñanza-aprendizaje para el estudiante inserto en estas modalidades a distancia. Aunque en este tipo de modalidades el aprendizaje se remite al individuo que aprende, en tanto que es él quien incorpora las experiencias nuevas a partir de sus percepciones, conocimientos, sensaciones, etcétera, se trata, ante todo, de un proceso social mediante el cual el sujeto que aprende logra incorporar nueva información a través del contacto con otras personas, por lo que el aprendizaje se da cuando nos comunicamos personalmente.

Hasta aquí hemos considerado diferentes teorías que apoyan al aprendizaje dentro de una modalidad a distancia; sin embargo, resumimos estas aportaciones en el Cuadro 1, y explicamos cómo apoyan a la modalidad a distancia en bibliotecología, basándonos en el constructivismo, pero apoyándose también en otras teorías pedagógicas que sustentan la calidad de las modalidades a distancia en bibliotecología. 
Cuadro 1. Teorías que apoyan la modalidad a distancia

\begin{tabular}{|l|l|l|}
\hline \multicolumn{1}{|c|}{ Teoría } & \multicolumn{1}{|c|}{ Autor } & \multicolumn{1}{c|}{ Como apoya a la MAD ${ }^{12}$} \\
\hline $\begin{array}{l}\text { Aprendizaje } \\
\text { independiente. }\end{array}$ & Piaget & $\begin{array}{l}\text { El aprendizaje es el resultado de una construcción de es- } \\
\text { tructuras intelectuales a partir de materiales tomados de } \\
\text { la cultura, y en donde el estudiante adopta y genera las } \\
\text { destrezas y actitudes requeridas para su formación. }\end{array}$ \\
\hline $\begin{array}{l}\text { Teoría de la } \\
\text { comunicación. }\end{array}$ & $\begin{array}{l}\text { Barbero, Prieto, } \\
\text { Gutiérrez y Vigotskyy }\end{array}$ & $\begin{array}{l}\text { Para estos autores la conducta de los seres humanos así } \\
\text { como, el conocimiento están mediados por la sociedad } \\
\text { y la cultura. }\end{array}$ \\
\hline Neoconductivismo. & Skinner & $\begin{array}{l}\text { Fomenta la habilidad en el manejo y manipulación de } \\
\text { máquinas (computadoras, faxes, lectores, etcétera.); y } \\
\text { promueve la memoria por rutina para el aprendizaje de } \\
\text { ciertas habilidades motrices. }\end{array}$ \\
\hline Psicología humanista & Bates & $\begin{array}{l}\text { Para él, el conocimiento es construido por cada individuo } \\
\text { mediante la interpretación y comprobación del significa- } \\
\text { do de sucesos externos; es decir las experiencias pasa- } \\
\text { das de cada individuo. }\end{array}$ \\
\hline Cognoscitivista & Hernández Rojas. & $\begin{array}{l}\text { Aquí el procesamiento de la información consistió en } \\
\text { crear estudiantes críticos, reflexivos y que sepan solucio- } \\
\text { nar problemas. }\end{array}$ \\
\cline { 2 - 4 } & Bruner & $\begin{array}{l}\text { Para él, el aprendizaje se descubre y el currículo sirve } \\
\text { para pensar. }\end{array}$ \\
\cline { 2 - 4 } & Ausubel & El aprendizaje debe ser significativo y por asimilación. \\
\hline
\end{tabular}

\section{Ambientes y espacios de aprendizaje}

La importancia de la comunicación, como se vio con anterioridad, es sustancial para el aprendizaje, pero también lo es la relevancia que tiene el medio que rodea al individuo para incorporar mejor y más fácilmente conocimientos nuevos a los ya obtenidos con anterioridad. Es fundamental crear ambientes de aprendizaje en los cuales se generen espacios de interacción de ideas y conocimientos, espacios que pueden ser:

- Espacios de gestión.

- Espacios de prácticas (catalogación, clasificación, encabezamientos, etcétera).

- Espacios de conocimiento.

- Espacios de colaboración.

- Espacios de asesoría. 
Estos espacios no sólo permitirán generar un ambiente propicio para el aprendizaje sino que conformarán un entorno idóneo en el que se relacionan entre sí sujetos y objetos. Como sostiene Nassif:

el ambiente involucra la totalidad de las circunstancias externas al individuo o a las comunidades que actúan como estímulos sobre los mismos y ante los cuales reaccionan, se adaptan, responden o mueren; ${ }^{13}$

es decir, el ambiente se integra por los espacios y los objetos contenidos en ellos.

Asimismo recordemos que las Bibliotecas Digitales (BD) pueden considerarse objetos de aprendizaje, sobre todo si se observa sus contenidos, como espacios de aprendizaje, y si los percibimos como servicios para la educación a distancia. Así, si para los estudiantes de una modalidad presencial son importantes las BD, para los involucrados en una modalidad a distancia son fundamentales para obtener contenidos relevantes y elevar la calidad de su formación profesional, lo que los obliga a adoptar un papel responsable, no sólo como receptores pasivos de lo generado por el docente, sino como agentes activos en la búsqueda, selección, procesamiento y asimilación de la información.

Los elementos que se han mencionado con anterioridad, así como otros que se expondrán en las dos etapas siguientes, no se explican en su totalidad debido a que es fácil entender la aportación e implicación que tienen con los otros elementos en las modalidades a distancia. ${ }^{14}$

\section{ETAPA DE IMPLEMENTACIÓN}

La etapa de implementación debe de constar como mínimo de los siguientes elementos:

- Un modelo educativo.

- Los recursos tecnológicos que permitan la interacción y comunicación entre los diferentes involucrados en el proceso de enseñanza-aprendizaje de la modalidad.

13 Nassif citado por Manuel Moreno Castañeda. et.al. Desarrollo de ambientes de aprendizaje en educación a distancia, Guadalajara, Coordinación de Educación Continua Abierta y a Distancia de la Universidad de Guadalajara, 1998.

14 Los temas no se abordan a profundidad durante el presente artículo debido al límite de extensión pero se desarrollan ampliamente en el capítulo 3 de la tesis de doctorado. Cfr.: Brenda Cabral Vargas. La educación a distancia en bibliotecología, México, La autora, 2008. 
- Contenidos y planeaciones didácticas.

- Materiales didácticos.

- Docentes con el perfil requerido desde la planeación.

- Instrumentos de evaluación acordes con éste tipo de modalidad.

- Centros de asesorías ubicados en diferentes puntos geográficos.

Si bien en ésta etapa es donde pondremos en acción todo lo previsto durante la planeación, es también aquí donde nos daremos cuenta de todos los problemas y elementos no considerados en la etapa anterior. Por eso es necesario ir documentando qué elementos son excesivos, cuáles carentes y cuáles insuficientes, con la finalidad de que al llegar a la etapa de consolidación se puedan implementar procesos de mejora que permitan lograr la calidad de cualquier modalidad a distancia en bibliotecología.

Pero aunque los espacios hayan sido concebidos desde la etapa de la planeación, es en la etapa de implementación cuando se tiene que ir supervisando la funcionalidad de dichos espacios dentro del proceso de aprendizaje, y cuando hay que tener presente el modelo educativo en el que se basa el plan de estudios.

\section{Recursos tecnológicos y de comunicación}

Por lo tanto se hace necesario pensar en el impacto de la tecnología, donde los medios electrónicos y de información son el espacio de producción de significados y consensos a través de la mediación de los programas de estudio o trabajo, y las organizaciones grupos e individuos, considerando que no todo es "[...]mentalidad tecnológica ni todas las innovaciones educativas pasan por los microchips"; ${ }^{15}$ los medios escritos no virtualizados juegan aquí sin duda un importante papel. Al igual que la experiencia que, sin descartar la influencia genuina de la tecnología en el rendimiento socio-intelectual, seguirá teniendo una importante función en el desarrollo de la inteligencia.

Si en la etapa de planeación se realizó de manera adecuada la descripción de la infraestructura tecnológica y de comunicación, se tendrá claro en esta otra la descripción de perfiles, funciones y antecedentes del personal a cargo del área tecnológica, lo que garantizará los soportes puestos a disposición del programa, sus niveles de operación y su confiabilidad, así como los perfiles y la capacitación de los docentes en este aspecto, lo cual apoyará la transmisión de conocimientos de la asignatura en la cual son expertos. 


\section{Contenidos educativos}

El diseño y elaboración de contenidos se debe llevar a cabo desde la etapa de la planeación porque hay que considerar una serie de actividades, procesos y personas, aunque esto varía de una institución educativa a otra, en tanto que depende de sus políticas internas. Es en la etapa de implementación en la que se utilizarán los contenidos y por ello será hasta ese momento cuando detectemos sus fortalezas y debilidades a través de los comentarios emitidos, tanto por los asesores como por los estudiantes.

Se debe prever, además, que los materiales educativos de la modalidad a distancia sean productos comunicativos integrados por contenidos informativos, así como por actividades de aprendizaje que usen la información de estos contenidos. El educando deberá ir seleccionando e interactuando para generar su propio aprendizaje, co-aprendiendo con otros estudiantes y con su asesor. Dichos materiales se pueden encontrar en cualquier medio: impresos, en disco compacto, audiovisuales, plataformas electrónicas, casets, etcétera.

Hoy requerimos de cualquier tipo de soportes que puedan crear, a su vez, formas específicas de comunicación para lectores de documentos electrónicos. Porque el proceso de pensamiento cambia y deja de ser lineal, y les presenta a los lectores infinidad de posibilidades de lectura que evidencian una determinada dimensión conceptual requerida para su construcción. Si la construcción es eficiente, la ruta para la lectura de un documento se incrementa. A su vez, el lector puede fortalecer sus procesos mentales mediante la interpretación requerida para el análisis discursivo que se halla implícito en este tipo de construcción textual.

Asimismo el aprendizaje significativo, de acuerdo con el trabajo de Ausubel, se genera en dos dimensiones: la primera se refiere a la manera en que el estudiante incorpora la información a su esquema cognitivo, el cual puede ser repetitivo o memorístico o bien significativo, el cual implicaría recepción y descubrimiento; la segunda dimensión se refiere al tipo de estrategia o metodología de enseñanza empleada para que dicho aprendizaje se genere. Dentro de este último aspecto se señala que para que ocurra el aprendizaje significativo es necesario que el material posea significatividad lógica o potencial, y que entre el material de aprendizaje y los conocimientos previos de los alumnos exista una distancia óptima, para que éstos puedan encontrarle sentido; y también debe existir disponibilidad, intención y esfuerzo por parte del alumno para aprender. ${ }^{16}$

16 Greta Trangay Vázquez, Instrumentación de la modalidad de educación a distancia en la Licenciatura en Derecho y Ciencias Sociales de la Universidad Michoacana de San Nicolás Hidalgo, Michoacán, La autora, 2002, Tesis (Maestría en pedagogía), UNAM, FFYL, p.45. 
Si se cumple con estas tres condiciones

[...] la información podrá ser adquirida de forma sustancial [...] y no arbitrariamente; esto es, el estudiante, podrá relacionar semánticamente el material de aprendizaje con su conocimiento previo y, entonces, aprenderlo con comprensión atribuyéndole significado y sentido personal. ${ }^{17}$

Los audiovisuales y gráficos son parte esencial del patrimonio cultural de cualquier país, ya que éstos contienen una enorme cantidad de información que se debe dar a conocer y preservar para las generaciones futuras.

La educación a distancia debe retomar como material o recursos didácticos, fotografías, tarjetas, materiales de audio, música, videos, filmes o dvd, porque contienen información esencial que a veces los materiales monográficos o hemerográficos no tienen.

Un elemento indispensable en la etapa de implementación de las dichas modalidades es hacer más hincapié en la motivación, ya que es más fácil que por la distancia y por no tener una interacción directa con el docente y sus compañeros, el estudiante pueda desanimarse fácilmente; por eso es necesario echar mano de otros recursos como imágenes y sonidos: para despertar y fomentar el interés en las diferentes asignaturas; además de que la motivación tiene que ver con los problemas afectivos que afectan la trayectoria escolar (véase anexo 1).

\section{Docentes con el perfil requerido desde la planeación}

Pero todo lo anterior sería imposible si no contáramos con una serie de personajes que apoyen todas las actividades llevadas a cabo en una modalidad a distancia; a continuación mencionaré quienes serán esos recursos humanos y qué papel estarán llevando a cabo en éste tipo de modalidades:

- Los bibliotecarios nos acercarán a las fuentes y recursos digitales necesarios para argumentar los contenidos de los materiales.

- Los pedagogos se encargarán de la parte didáctica y metodológica del proceso de enseñanza- aprendizaje.

- Los informáticos o programadores se encargarán del soporte del software y hardware y facilitarán así el manejo de la información en la red.

- Los diseñadores gráficos serán los encargados de que los contenidos queden plasmados con textos, gráficos y diagramas de fácil interpretación que agilizarán el acceso a los contenidos. 
- Psicólogos; es fundamental la participación de estos profesionistas ya que son éstos quienes pueden proporcionar los elementos apropiados para que el alumno, a pesar de la distancia del docente, se sienta integrado y motivado para estudiar y profesionalizarse y no caiga en el desinterés por la falta de interacción con el docente o sus compañeros.

- Lingüistas; es importante la labor de dichos profesionales para que la educación a distancia se erija como una modalidad bien consolidadada de una sociedad del conocimiento, pues

[...] se requiere considerar la historia de las prácticas educativas y observar en ellas, la forma como se han valorado las competencias comunicativas. Estas competencias son el factor humano que generalmente se ha soslayado desde una visión de la modalidad a distancia, esencialmente informacional y tecnocrática. ${ }^{18}$

Es fundamental, además de los expertos antes mencionados y que participan para que funcione de manera adecuada una modalidad a distancia, considerar que los expertos en bibliotecología o ciencias afines, además de conocer la teoría, cuenten con la experiencia necesaria en la práctica profesional, pero además que conozcan los aspectos metodológicos, como los tecnológicos, que están tan íntimamente relacionados con este tipo de modalidad educativa.

\section{Alumnos}

En diversos apartados nos hemos referido al alumno y a su acercamiento al aprendizaje, los contenidos, las teorías y los diversos expertos involucrados en la enseñanza a distancia; también es primordial que conozcamos su trayectoria académica para poder solucionar sus problemas (véase anexo 1).

\section{Instrumentos de evaluación acordes con éste tipo de modalidad}

En esta etapa, la evaluación es una actividad que nos permite conocer qué tanto ha asimilado o comprendido los conocimientos un estudiante. Dicho de otra manera se trata de estimar los conocimientos, actitudes, aptitudes y rendimiento de un alumno.

18 María Elena Chan Núñez, Los ambientes y materiales en el diseño de ambientes de aprendizaje en la educación a distancia, En El medio digital en el siglo XXI : retos y perspectivas para los bibliotecólogos, investigadores, educadores, y editores [Disco Compacto], Angélica Rosas Gutiérrez, comp. Georgina Araceli Torres Vargas, México, CUIB, 2001. 
Existen varias formas o métodos para poder evaluar; sin embargo, veremos a continuación cómo el constructivismo puede ayudar a este tipo de modalidad, ya que concretamente en la educación a distancia tenemos que rescatar los principios que la orientan, pues la conjunción de éstos con los de la evaluación constructivista identificarán las posibilidades de la modalidad educativa que nos ocupa.

Recordemos algunos de los principios de la educación a distancia que es sustancial subrayar en esta modalidad el estudiante es un sujeto con determinadas características de independencia, autogestión y responsabilidad, y además de que el objetivo de la educación a distancia es lograr aprendizajes significativos en el marco de la postura constructivista, debemos pensar también en la instrumentación de una evaluación acorde con dicha modalidad.

Kelly (1970: 1), remarcó:

sea lo que sea lo que acabe pasando con la búsqueda de la verdad, los acontecimientos con los que nos enfrentamos hoy están sujetos a tanta variedad de construcciones como nuestro ingenio nos permita inventar. Esto no quiere decir que cualquier construcción es tan buena como otra[..]. Pero nos recuerda que todas nuestras percepciones presentes están abiertas al cuestionamiento y la reconsideración, y sugiere ampliamente que incluso las ocurrencias diarias podrían aparecer completamente transformadas si tuviéramos imaginación suficiente para construirlas de modo diferente. ${ }^{19}$

Lo expresado por Kelly, invita a reflexionar en que se le deben propiciar al sujeto situaciones de aprendizaje y evaluación en las que se transformen los pasos y las secuencias de todo proceso, en las que se reconstruyan situaciones y posibles resultados; y todo aquello que permita la creatividad, pero sobre todo el análisis y el cuestionamiento sobre cómo se resuelven o cómo se realizan los procesos.

Por lo anterioriormente expresado es necesario que las estrategias de evaluación sean constructivistas, como ejemplo podemos mencionar algunas formas que se utilizan con dicho fin, como la entrevista interactiva en lugar de los cuestionarios prefabricados. Algunos estilos de entrevista son: el escalamiento o flecha descendente; en la primera, como su nombre lo indica, se va ascendiendo en el tratamiento de un asunto o problemática, con la intención de que esto permita ampliar el conocimiento y, sobre todo, reconocer lo que se sabe en torno a algo en concreto; en el caso de la flecha descendente, se pretende la profundización para encontrar el origen o las causas de un problema. 
Otra técnica de evaluación constructivista es el denominado Análisis del Discurso, por la importancia que reviste para su utilización en la educación a distancia.

Esta técnica implica el seguimiento de grupos de estudio, con los que, pese a la distancia, se pueden instrumentar mecanismos de interacción y encuentros presenciales, sobre todo a través de medios tales como la teleconferencia, la audioconferencia, los foros de análisis y discusión vía Internet, etcétera. Lo esencial para el desarrollo de la técnica es el tipo de abordaje que se realice de los asuntos o tópicos, lo que deberíamos buscar son formas que les permitan a los involucrados una participación activa y la identificación de los obstáculos en su proceso de aprendizaje.

El docente en la educación a distancia deja de ser aquél que transmite los conocimientos y también el mediador entre los alumnos y los contenidos disciplinarios, los cuales están inmersos en los propios cursos, y pasa a convertirse en un evaluador permanente del aprendizaje de cada uno de sus estudiantes, analizando y valorando las ejecuciones para consolidar y reorientar los logros. En este sentido para la educación a distancia la evaluación continua del aprendizaje es esencial, y por ende resulta conveniente que se base en diferentes instrumentos que permitan apreciar el avance de cada alumno en los distintos niveles y tópicos por los que transita al adquirir el conocimiento. Es trascendental por ello llevar además un buen control de su trayectoria académica, lo que permitirá darle solución a algunos de los problemas que afectan dicha trayectoria, (véase anexo 1).

En el 2006 Quesada ${ }^{20}$ mencionó que son cuatro los principios fundamentales de la evaluación del aprendizaje, los mencionamos a continuación:

- Confiabilidad; lo observado en los instrumentos empleados debe reflejar exactamente el logro de los estudiantes. En el futuro, el grado de confiabilidad que tengan los instrumentos de evaluación del aprendizaje será uno más de los estándares para juzgar el curso.

- Validez; el instrumento elegido debe reflejar lo que se conoce como "dominio" del tema, aludiendo no al grado de destreza que se ha alcanzado, sino a su representación adecuada para que unas cuantas actividades o preguntas manifiesten si se sabe, o si se sabe hacer lo que se busca. Seguramente este principio también formará parte de los estándares requeridos para los cursos a distancia, "[...] los instrumentos de

20 Rocío Quesada Castillo, Evaluación del aprendizaje en la educación a distancia [En línea], no.16 (30 sept. 2006) en RED. Revista de Educación a Distancia. (Número especial dedicado a la evaluación en entornos virtuales de aprendizaje), Disponible en: http://www.um.es/ead/ red/M6, (consulta: 22 de octubre del 2006). 
evaluación del aprendizaje deben probar su validez, sobre todo la de constructo, contenido y aparente". ${ }^{21}$

- Objetividad; la objetividad forma parte integrante de cualquier evaluación. De nada serviría juzgar cualquier mérito si lo que fundamenta la calificación obtenida es el favoritismo, el prejuicio, la corrupción o cualquier elemento ajeno al atributo y ejecución que se evalúa. Dentro de los estándares para valorar un curso a distancia debiera considerarse el análisis sobre la forma en la cual sus instrumentos de evaluación del aprendizaje aseguran la objetividad.

- Autenticidad; la evaluación del aprendizaje debe ser auténtica; esto es, debe manifestar que los procesos intelectuales que se ponen en juego en ella corresponden a aquellos que el alumno usará en las situaciones reales de aplicación del conocimiento en cuestión, como mencionaron Chacón, ${ }^{22}$ 1994; Honebein, ${ }^{23}$ Duffy y Fishmann en 1994; y Morgan y O’Reilly, 1999.

Tres son las funciones de la evaluación del aprendizaje a distancia y éstas son: diagnóstica, formativa y sumativa; las cuales de manera integral permiten ver cada una de las fases de conocimiento por las que va pasando el estudiante.

Ahora bien los instrumentos más utilizados para evaluar los aprendizajes en una modalidad a distancia son: la prueba objetiva, las preguntas intercaladas, las pruebas adaptativas y autoadaptadas, la prueba de ensayo, el proyecto, el interrogatorio, la lista de verificación, las escalas, la rúbrica, el portafolio y el mapa conceptual.

A manera de resumen de este apartado podemos mencionar asegurar los siguientes aspectos:

- El tutor funge como evaluador permanente del aprendizaje del alumno.

- A lo largo de todo el curso la evaluación formativa es una más de las actividades de enseñanza.

- Las actividades que realiza el alumno se convierten en espacios de evaluación.

- La evaluación del aprendizaje se individualiza.

- El peso que la evaluación tiene en la formación obliga a una planeación

21 Universidad Virtual de Michigan. 2002, [en línea] Standards for quality online courses, [consulta: 24 de octubre del 2006], Disponible en: http://www.um.es/ead/red/M6/quesada.pdf

22 Chacon, Idem.

23 P. Honebein, Duffy, T. y Fishman, B. "Constructivism and the design of learning environments; context and authentic activities for learning", en Designing environments for constructive learning, Heidelberg, Springer Verlag, 1994, 374 p. obra completa. 
y ejecución cuidadosas y al análisis atento de cada uno de sus elementos.

- Cada evaluación conlleva una retroalimentación inmediata y permite la inclusión de actividades remediales.

Las nuevas modalidades de evaluación de los aprendizajes se orientan a valorar la significatividad de éstos, lo cual representa una tarea de gran complejidad. Como se ha expuesto, este tipo de aprendizaje es una actividad progresiva y sólo puede ser evaluada cualitativamente.

El docente debe tener claridad respecto al grado de apropiación con el que intenta aprenderse algún contenido. Los objetivos deben formularse explícitamente y deben establecer la extensión o amplitud y el nivel de complejidad con que el estudiante ha elaborado esquemas o significados con apoyo del docente. Estas características obligan a asociar de manera estrecha las actividades de enseñanza, aprendizaje y evaluación.

\section{Centros de asesorías ubicados en diferentes puntos geográficos}

La estructura orgánica es un elemento que no se puede olvidar puesto que permite descentralizar las asesorías, y tener un mejor acercamiento e interacción más cercana con los estudiantes. Al analizar dos modelos de educación bibliotecología de dos países de América Latina (Cuba y Argentina), independientemente del modelo que manejan, y que se muestra en el Cuadro del anexo 2 , ambos consiguen a través de la creación de centros de apoyo en diferentes puntos geográficos, un mayor control y motivación en los estudiantes y de este modo logran una mayor eficiencia terminal.

\section{Etapa de Consolidación}

Se abordarán ahora otros elementos primordiales que deben incluirse en las modalidades de educación a distancia, y que hay que considerar en la última etapa, la de consolidación. Éstos son:

- Los cuerpos académicos.

- La investigación.

- La calidad educativa (indicadores)

Al ver estos tres conceptos muchos podrían cuestionarse si realmente una modalidad a distancia requiere de dichos elementos; respondiendo a ello 
explicaremos que los cuerpos académicos son importantes para cualquier institución de educación superior, ya que a partir de ellos se puede medir la solidez de la educación a distancia y su capacidad para cumplir con eficacia los propósitos institucionales, lo que sólo puede concebirse mediante el fortalecimiento de la calidad y participación de sus cuerpos académicos.

Los cuerpos académicos pueden definirse como grupos de docentes que tienen:

- Intereses y objetivos académicos coincidentes.

- Objetos de investigación comunes.

- Formas de producción y transmisión del conocimiento compartidas (particulares de algún campo, en nuestro caso, sería el bibliotecológico). ${ }^{24}$

Los cuerpos académicos permitirán mejorar los ambientes académicos, ayudarán a cumplir con los objetivos institucionales; además de ser la fuerza motora del desarrollo institucional que servirá a la autorregulación del funcionamiento de la educación a distancia.

El impacto institucional de los cuerpos académicos puede girar en dos vertientes:

El interno que tiene que ver propiamente con la docencia, y en el cual se reflejará la aceptación que tendrán los egresados en el mercado laboral y la aceptación de dichos egresados en los programas de postgrado de excelencia.

Y el impacto externo, que se verá reflejado propiamente en la investigación, en los siguientes aspectos:

- Aceptación de sus trabajos en los medios prestigiosos de publicación en la disciplina.

- Impacto de sus resultados en la solución de problemas del entorno.

- Impacto de sus resultados en la comunidad científica (referencias, citas o patentes).

- Participación en las redes y grupos especializados.

Con el análisis de este apartado se percibió que la labor de los cuerpos académicos en cualquier modalidad a distancia de educación superior, es prescindible para lograr un mejor desempeño en diversos aspectos de las actividades docentes de dicha modalidad, así como lograr desarrollar investigación y difusión del conocimiento en diversas áreas del conocimiento humano.

24 Magdalena Fresan Orozco, Los cuerpos académicos [diapositivas en línea] México, ANUIES, 2000, Disponible en: http://www.anfei.org.mx/merida1.pdf (Consulta: 11 de mayo del 2006) p. 4 
Ahora bien la investigación en nuestra disciplina es uno de los pilares de la educación, ya que con ella se pretende a través de una búsqueda, ya sea de campo o bibliohemerográfica, encontrar la respuesta a varios problemas presentados en ella, además de fundamentar y complementar a la bibliotecología con bases teóricas.

La investigación en las modalidades de educación a distancia en nuestros países es casí nula, la anterior aseveración quedo demostrada a través de un estudio llevado a cabo por Marisol Ramírez del ITESM, Campus Juárez en la relación entre la investigación y la educación a distancia, se observaba que la gran mayoría de los programas de educación a distancia no fomentan la investigación, actividad tan importante dentro de la educación superior. De hecho, el estudio de Ramírez señalaba que el $83 \%$ de las modalidades de educación a distancia no efectuaban investigación, y que sólo la efectuaba un $17 \%$ como parte de su formación ${ }^{25}$. Debido a lo anterior debemos intentar incrementarla, lo que quizás podría promoverse ayudándonos con la biblioteca digital, su bibliografía y sus espacios de aprendizaje.

La biblioteca digital podría servir para fomentar la investigación en las instituciones educativas que tienen la modalidad de educación a distancia de la siguiente manera:

- Difundir artículos o textos interesantes de interés para sus usuarios.

- Establecer foros de discusión sobre temas de interés para el área.

- Fomentar la colaboración e integración de equipos de trabajo con sus usuarios y con algunos expertos o centros que manejen temas afines.

- Permitir que algunos servicios de información manden sus servicios de alertas o que puedan difundir sus investigaciones a través de los títulos de revistas generados por dichas empresas.

La calidad es un aspecto que tendría que considerarse desde la planeación para que todas las acciones se enfoquen a alcanzar este fin. Esta calidad se puede medir de varias maneras, pero al respecto Cookson comenta que:

[...] la excelencia puede definirse como el estado de ser bueno o superior de acuerdo con ciertas normas. En el campo de la educación abierta y a distancia los estándares pueden ser establecidos por instituciones, asociaciones o agencias. En la educación superior en general, el concepto de la calidad se refiere a la atención de las tecnologías en la educación a distancia. México : ITESM, 2002. 
a los clientes, la coherencia en los procesos de enseñanza y aprendizaje, y la capacidad de respuesta de las necesidades siempre cambiantes de los clientes. ${ }^{26}$

Para conocer si las modalidades a distancia han incrementado la calidad en la educación es necesario contar con indicadores, pero antes de desarrollar éstos es preciso plantearnos la siguiente pregunta ¿qué es un indicador? Algunos autores (Alicia Pinzas, Eudoro Terrones Negrete, Janice Miller y Claire Bahamon ${ }^{27}$ lo definen como un dato estadístico que permite elaborar un juicio sobre el funcionamiento de un proceso, sistema o Institución. En nuestro caso consideraremos a los indicadores, como elementos informativos de control que nos permitan conocer cómo funciona una cierta actividad, pues hacen referencia a parámetros estables que sirven para establecer la magnitud de comprobación acerca del funcionamiento de alguna actividad en particular.

Los indicadores pueden ser tanto cuantitativos como cualitativos, aquí utilizaremos ambos tipos porque en algunos casos mediremos cambios en cantidad (como el aprovechamiento escolar, la deserción o la productividad), y en otros se considerarán aspectos que se relacionan más con lo cualitativo, como valores, conceptos o el saber, que intervienen en un sistema académico: la satisfacción, la honestidad el compromiso, el involucramiento y el respeto.

\section{Características de los indicadores.}

Para que dichos indicadores puedan realmente fungir como elementos de control y de información tendrán que ser fácilmente medibles e interpretables, libres de sesgo, objetivos, precisos, unívocos, sensibles a los cambios, repetibles a través del tiempo y analizables contra los otros indicadores.

Contar con indicadores permite, además de medir los elementos que interactúan en la educación a distancia, también entender mejor dichas relaciones y de esta manera poderlas controlar para finalmente mejorar la calidad en todas las actividades y procesos de este tipo de educación.

Si las modalidades de educación a distancia cuentan con indicadores de medición podrá lograrse un alto índice de efectividad en las acciones de mejora

26 P. S. Cookson, "Acceso y equidad en la educación a distancia: investigación, desarrollo y criterios de calidad", en Revista Electrónica de Investigación Educativa, 2002, 4 (2), Disponible en: http://redie.uabc.mx/vol4no2/contenido-cookson.html (consulta: el 15 de febrero del 2006)

27 Cfr.: Pinzás, Alicia. "Las mujeres, las palabras y el mundo global: Glosario". Lima, Centro de la Mujer Peruana Flora Tristán, 1995, p. 21; Terrones Negrete, Eudoro. "Diccionario de investigación científica”. Lima, A.F.A. Editores Importadores, 1998. p. 191; Miller, Janice y Calire Bahamon. "A pocket glosary in three languages". Massachusetts, Family Plannin Management Development - FPMD, 1995, p. 120. 
tomadas por la institución educativa involucrada. Y también se podrán establecer claramente a todos los niveles de esa institución cuales son los parámetros importantes a los cuales la dirección les dará seguimiento.

\section{Conclusiones}

Las teorías de enseñanza y aprendizaje son la base para diseñar un modelo educativo, que permita orientar y definir las funciones y relaciones entre los actores del proceso educativo y el papel que a cada uno se le asigna en tal proceso. Es a partir de la selección del modelo educativo que se elija, que se deriva el currículum tomando en consideración el funcionamiento de la estructura académico-administrativa.

La revisión de bibliografía y de programas en bibliotecología a distancia nos permitió conocer, pero también reflexionar, sobre la importancia de cada uno de los elementos incluidos en este tipo de modalidad, y la manera en que contribuyen al proceso de enseñanza-aprendizaje de los estudiantes que se encuentran insertos en esta modalidad educativa.

La importancia de difundir en este artículo el papel que desempeña cada uno de los elementos mencionados para las modalidades a distancia, les permitirá a las instituciones educativas interesadas en desarrollar estudios en bibliotecología a distancia, considerarlos a la hora de planear, implementar y evaluar una modalidad a distancia. De no hacerse así, puede surgir una serie de problemas que afectarían a los estudiantes y a la sociedad en general y no generarían un ambiente propicio para el aprendizaje de estos profesionales, con lo cual no se estaría formándolos con la pertinencia requerida para desempeñar sus actividades dentro de la fuerza laboral.

La posibilidad de compartir recursos humanos y materiales en las modalidades a distancia en la bibliotecología de América Latina, es remota aún, puesto que en la fase en la que se encuentran actualmente dichas modalidades, tanto a nivel nacional como regional, no existen todavía muchos elementos en común. Sin embargo, se pueden compartir experiencias de los resultados obtenidos a lo largo de la puesta en funcionamiento de algunos modelos educativos en esta modalidad en bibliotecología, y pueden también revisarse las fortalezas y debilidades de cada elemento incluido en ellas; así como las teorías y métodos que han tenido mejores resultados en esta modalidad educativa. Será sin duda necesario que los docentes en estas modalidades cambien sus actitudes habituales y obtengan conocimientos sobre las herramientas y estrategias de aprendizaje que necesita este tipo de modalidad 82 educativa. 
Entre nosotros hay docentes que aunque llevan años impartiendo cátedras en las modalidades presenciales y tienen mucha experiencia, no son aptos para este tipo de modalidad, debido a que su papel deja de ser únicamente el de transmisor del conocimiento. Hoy debemos concebir y practicar la enseñanza participando como actores innovadores y facilitadores del aprendizaje. Y por otro lado, los docentes tienen que involucrarse también en la creación de materiales de aprendizaje, como queda evidenciado en el Cuadro incluido en el anexo 2, y que muestra cómo los 3 casos analizados de instituciones educativas que tienen modalidad a distancia cuentan con materiales que responden a los métodos pedagógicos para transmitir los conocimientos. Igualmente, estos docentes tendrán que involucrarse en la utilización de tecnologías de educación a distancia y en la creación de ambientes de comunicación que permitan la interacción entre la institución educativa, el tutor, el estudiante y los responsables de los materiales didácticos, dentro de un plan de estudio que cuente con una planeación previa en cuanto a sus contenidos.

El material didáctico y las guías de aprendizaje comparten elementos característicos, como es el hecho de contar con un equipo multidisciplinario que se encarga de planear con precisión los procedimientos y tareas que deben llevarse a cabo. Pero ambos deben partir de un objetivo, además de considerar las teorías del aprendizaje para planear las actividades de aprendizaje.

Tener claros los elementos que intervienen en una modalidad a distancia permite conformar la base sobre la cual poder diseñar un modelo de educación a distancia acorde y pertinente para formar los profesionales que la sociedad requiere. Además es conveniente primero buscar y seleccionar los conocimientos teóricos relativos al proceso mismo de enseñanza-aprendizaje, los métodos, las formas y los elementos que deben conformar el modelo de educación a distancia en bibliotecología, pero además sería bueno contar con las experiencias adquiridas por algunas de las escuelas que han implementado sus modalidades a distancia en el área. Por ello también se debe

[...] dejar madurar la aplicación de teorías y metodologías propias del modelo de educación a distancia [...] el cual es un elemento que puede coadyuvar a la construcción de un nuevo paradigma para la enseñanza de la Bibliotecología y los Estudios de información. ${ }^{28}$

28 Noemí Conforti,. "Universidad Nacional de Mar del Plata : la educación a distancia en la formación de los bibliotecarios." En: Reunión Nacional de bibliotecarios (35 : 2001 : Buenos Aires, Argentina), Las bibliotecas, el ciudadano y el derecho a la información. Buenos Aires, Asociación de Bibliotecarios Graduados de la República Argentina, 2001., p.155. 
Es importante mantener la calidad como una prioridad durante las tres etapas, y el todo debe ser visto como un proceso continuo, sin olvidar que en la actualidad la mejora y el aseguramiento de la calidad de la educación es un aspecto que la gestión gubernamental está tomando muy en consideración; y que dependiendo de los resultados de ésta, se brinda financiamiento para mejorarla y asegurarla.

Promover e institucionalizar los procesos de acreditación y certificación de la calidad, impulsar la participación de sectores sociales que tradicionalmente han sido excluidos de la toma de decisiones públicas y darle nuevo énfasis a la evaluación como la plataforma que nos ayudará a elevar la calidad, son algunas de las aristas que se han introducido en el quehacer gubernamental e institucional de la educación en nuestro país; de manera especial y con énfasis acentuado en la educación superior. La calidad tiene que ser considerada ahora como un factor primordial, y debemos planear, desarrollar y consolidar la modalidad a distancia en bibliotecología considerando el modelo propuesto. Sin embargo, es necesario concientizar a las autoridades educativas de que la calidad no debe ser vista únicamente como un requisito para obtener la acreditación y la certificación, lo que hace falta es tener una visión más amplia encaminada hacia la pertinencia y excelencia en la formación de los futuros bibliotecólogos, con la finalidad de ir mejorando y fortaleciendo la disciplina para que ésta pueda satisfacer las expectativas que han sido puestas en ésta.

\section{OBRAS CONSULTADAS}

Bustamantes Rojas, H.G. Propuesta de un modelo de orientación educativa a distancia. En: Educación y formación a distancia. Práctica, propuestas y reflexiones. México: Universidad de Guadalajara, 2001.

Chacon, F. Un modelo de evaluación de los aprendizajes en educación a distancia. [En línea]. Conferencia Magistral de la Primera Reunión Latinoamericana de Educación a Distancia, 1994. Disponible en: www.anep.edu.uy/webct/oferta_edu/exp_contenidos/swf/ pg/lect/14/Lectura5.doc (Consulta: el 24 de noviembre del 2006)

Chan Núñez, María Elena, Los ambientes y materiales en el diseño de ambientes de aprendizaje en la educación a distancia. En: El medio digital en el siglo XXI : retos y perspectivas para los bibliotecólogos, investigadores, educadores, y editores [Disco Compacto] / Angélica Rosas Gutiérrez, comps. Georgina Araceli Torres Vargas. México : CUIB, 2001. 
Colom Canellas, A.J. Pedagogía tecnológica para la educación a distancia. En los nuevos escenarios y las transformaciones tecnológicas,

Conforti, Noemí. "Universidad Nacional de Mar del Plata : la educación a distancia en la formación de los bibliotecarios." En: Reunión Nacional de bibliotecarios (35 : 2001 : Buenos Aires, Argentina). Las bibliotecas, el ciudadano y el derecho a la información. Buenos Aires: Asociación de Bibliotecarios Graduados de la República Argentina, 2001. [pág. varía].

Cookson, P. S. Acceso y equidad en la educación a distancia: investigación, desarrollo y criterios de calidad [en línea]. Revista Electrónica de Investigación Educativa, 2002. v. 4 no.2. (Consulta: 15 de febrero del 2006) Disponible en: http://redie.uabc.mx/vol4no2/ contenido-cookson.html

Encuentro de directores y encuentro de docentes de escuelas de bibliotecología y ciencias de la información $\left(7^{\circ}\right.$ y $6^{\circ}: 2004$ : Mar de la Plata, Argentina.) Argentina : MERcosur, 2005 [En línea] Disponible en: http://www.mdp.edu.ar/humanidades/extension/ebcim04/archivos/trabajos.htm (consulta: 17 de mayo del 2006)

Fresan Orozco, Magdalena. Los cuerpos académicos [diapositivas en línea] México : ANUIES, 2000?. (Consulta: 11 de mayo del 2006) Disponible en: http://www.anfei.org.mx/merida1.pdf

Garduño Vera, Roberto. Modelo para la enseñanza vía internet de una especialización en organización de recursos informativos digitales. Madrid : el autor, 2002. 2 v. Tesis (Doctorado en ciencias de la información) - Universidad Complutense de Madrid. Facultad de Ciencias de la Información, Departamento de Biblioteconomía y Documentación.

Hernández R., Gerardo. "Proyecciones de aplicación del paradigma cognitivo al contexto educativo" En: Paradigma en psicología para la educación. Piados-educador, 1997.

Intec. Programas Académicos [En línea]. Disponible en: http://www. intec.edu.do/programas/hum/bci.html (consulta: 12 de septiembre de 2007)

Miller, Janice y Calire Bahamon. A pocket glosary in three languages. Massachusetts, Family Plannin Management Development FPMD, 1995

Moreno Castañeda, Manuel. et.al. Desarrollo de ambientes de aprendizaje en educación a distancia. Guadalajara : Coordinación de Educación Continua Abierta y a Distancia de la Universidad de Guadalajara, 1998.

Neimeyer Greg, J. (Comp.). Evaluación Constructivista. [Tr: Angela Aparicio]. Buenos Aires, Argentina : Paidós, 1996. 224 p.

Piaget, J. Seis estudios de psicología. México : Planete agustini, 1993. $225 \mathrm{p}$. 
Pinzás, Alicia. "Las mujeres, las palabras y el mundo global: Glosario”. Lima, Centro de la Mujer Peruana Flora Tristán, 1995.

Quesada Castillo, Rocío. Evaluación del aprendizaje en la educación a distancia [En línea]., no.16 (30 sept. 2006) En: RED. Revista de Educación a Distancia. (Número especial dedicado a la evaluación en entornos virtuales de aprendizaje). Disponible en: http://www. um.es/ead/red/M6 (Consulta: 22 de octubre del 2006).

Ramírez, Marisol. Acercamientos a los ambientes virtuales en México : Investigación sobre el uso de las tecnologías en la educación a distancia. México : ITESM, 2002.

Skiner, B. Ciencia y conducta Humana. España: Editorial Fontanela, 1974.

Terrones Negrete, Eudoro. Diccionario de investigación científica. Lima, A.F.A. Editores Importadores, 1998.

Trangay Vazquez, Greta. Instrumentación de la modalidad de educación a distancia en la Licenciatura en Derecho y Ciencias Sociales de la Universidad Michoacana de San Nicolás Hidalgo. Michoacán : La autora, 2002. - Tesis (Maestría en pedagogía) - Universidad Michoacana de San Nicolás Hidalgo.

Universidad Virtual de Michigan. 2002. [en línea] Standards for quality online courses. [Consulta: 24 de octubre del 2006] Disponible en: http://www.um.es/ead/red/M6/quesada.pdf

Vigotsky, L. S. Obras escogidas tomo I, Visor, Madrid, 1991. 


\section{ANEXO 1}

El siguiente anexo permite conocer cual es el conjunto de problemas que afectan la trayectoria escolar de los estudiantes a distancia, tal conjunto puede agruparse y jerarquizarse en cuatro tipos, en los que se conjugan elementos relativos a los estudiantes, su ambiente socio cultural y familiar, los docentes, los programas y los servicios administrativos y de apoyo académico de la escuela:

\begin{tabular}{|c|c|}
\hline Académicos & $\begin{array}{l}\text { 1. Antecedentes escolares, regularidad en la trayectoria escolar, rendimiento académico. } \\
\text { 2. Conocimientos básicos: historia de las culturas, técnicas de investigación documen- } \\
\text { tal, método científico. } \\
\text { 3. Competencias comunicativas, manejo del lenguaje, habilidades de lectoescritura, } \\
\text { hábitos de lectura. } \\
\text { 4. Competencias informativas: búsqueda, valoración, organización y aplicación de información } \\
\text { 5. Espacios curriculares para el desarrollo de hábitos y habilidades adecuados para el } \\
\text { desarrollo de trabajos de investigación. } \\
\text { 6. Expectativas ante la formación profesional: conocimiento previo sobre los programas } \\
\text { educativos y el campo profesional. } \\
\text { 7. Perfil de los docentes que imparten los primeros cursos: ser motivadores, manejo del } \\
\text { PE, ser flexibles y críticos. } \\
\text { 8. Orientación escolar oportuna. } \\
\text { 9. Servicios y apoyos académicos oportunos y eficientes: biblioteca, laboratorios, archivos }\end{array}$ \\
\hline Afectivos & $\begin{array}{l}\text { 10. Auto imagen, manera en el estudiante se percibe. } \\
\text { 11. Autoestima, grado de satisfacción consigo mismo. } \\
\text { 12. Ambiente familiar: estabilidad o desavenencias en el núcleo familia, apoyos y estímu- } \\
\text { los afectivos y materiales a las actividades de los estudios, etc. }\end{array}$ \\
\hline Metodológicos ${ }^{29}$ & $\begin{array}{l}\text { 13. Hábitos de estudio. } \\
\text { 14. Estrategias de aprendizaje. } \\
\text { 15. Existencia de Líneas para la Generación y Aplicación del Conocimiento (LGAC). } \\
\text { 16. Espacios curriculares para el desarrollo de trabajos de investigación. } \\
\text { 17. Flexibilidad en el desarrollo de las LGAC. } \\
\text { 18. Asesoría académica adecuada. }\end{array}$ \\
\hline Socioculturales & $\begin{array}{l}\text { 19. Acceso a bienes y servicios culturales, } \\
\text { 20. Nivel de percepción y distribución de los ingresos familiares, } \\
\text { 21. Nivel de responsabilidad en la contribución al ingreso familiar. } \\
\text { 22. Compatibilidad entre horarios laboral y escolar } \\
\text { 23. Tiempos de traslado de su domicilio a la escuela. } \\
\text { 24. Acceso a becas, ayudas médicas, apoyos alimentarios. } \\
\text { 25. Programas de atención integral: deportivos, artísticos, recreativos. }\end{array}$ \\
\hline Administrativos & $\begin{array}{l}\text { 26. Información confiable y oportuna sobre la trayectoria escolar } \\
\text { 27. Duplicación, omisión u obsolescencias de procedimientos administrativos } \\
\text { 28. Desinformación sobre procedimientos académico administrativos. } \\
\text { 29. Subordinación de procesos académicos a los procesos administrativos } \\
\text { 30. Inflexibilidad de los proceso de asignación de docentes, tutores y asesores. }\end{array}$ \\
\hline
\end{tabular}

29 Entiendo la metodología como un conjunto articulado de elementos que permiten percibir un problema, organizar actividades que puedan resolverlo, un bagaje de recursos técnicos e instrumentales aplicables de manera pertinente, criterios para evaluar la efectividad de las decisiones y ejecuciones efectuadas, flexibilidad para modificar los procedimientos adecuados a las circunstancias en que se desenvuelve el proceso 
Los países elegidos a continuación se seleccionaron por contar con modelos diferentes, lo que nos permitirá conocer más de cerca las variedades y similitudes en programas enfocados a la formación del profesional de la información.

\begin{tabular}{|c|c|c|c|}
\hline Rubro & Cuba $^{30}$ & Argentina $^{31}$ & Puerto Rico \\
\hline Carrera & $\begin{array}{l}\text { Información } \\
\text { científico-técnica y } \\
\text { bibliotecológica }\end{array}$ & $\begin{array}{l}\text { Bibliotecario escolar a } \\
\text { distancia }\end{array}$ & $\begin{array}{l}\text { Escuela Graduada de Bi- } \\
\text { bliotecología y Ciencia de } \\
\text { la Información }\end{array}$ \\
\hline Nivel & Licenciatura & Licenciatura & Maestro bibliotecario \\
\hline Modelo & Modelo de tipo mixto. & $\begin{array}{l}\text { Modelo de comunicación } \\
\text { multidireccional. }\end{array}$ & $\begin{array}{l}\text { Está basado en un modelo } \\
\text { constructivista de apren- } \\
\text { dizaje. }\end{array}$ \\
\hline Estructura Orgánica & $\begin{array}{ll}4 & \begin{array}{l}\text { Centros de educación } \\
\text { superior territoriales. }\end{array} \\
6 & \begin{array}{l}\text { Centros de educación } \\
\text { provinciales. } \\
\text { Instituciones de aten- } \\
\text { ción provincial o mu- } \\
\text { nicipal. }\end{array} \\
\end{array}$ & $\begin{array}{l}16 \text { Centros Regionales de } \\
\text { educación abierta y } \\
\text { una sede permanen- } \\
\text { te Central. }\end{array}$ & $\begin{array}{l}\text { Está integrado por } 14 \\
\text { Universidades y colegios } \\
\text { de E. U. y Puerto Rico. }\end{array}$ \\
\hline $\begin{array}{l}\text { Características del plan } \\
\text { de estudios }\end{array}$ & $\begin{array}{l}\text { Se estructura en } 3 \text { ciclos } \\
\text { de disciplinas. }\end{array}$ & $\begin{array}{l}\text { Se estructuro en tres ejes } \\
\text { temáticos: } \\
\text { 1) Institución educativa } \\
\text { 2) Formulación de pro- } \\
\text { yectos en la bibliote- } \\
\text { ca escolar. } \\
\text { 3) La práctica profesio- } \\
\text { nal. }\end{array}$ & $\begin{array}{l}\text { Los cursos se seleccio- } \\
\text { narán por parte de los } \\
\text { estudiantes de acuerdo } \\
\text { con sus capacidades. }\end{array}$ \\
\hline Matrícula & $\begin{array}{l}\text { 5,000 estudiantes en to- } \\
\text { dos los centros. }\end{array}$ & 233 alumnos. & \\
\hline Material educativo & $\begin{array}{l}\text { Todas las asignaturas } \\
\text { están respaldadas bi- } \\
\text { bliográficamente por un } \\
\text { programa analítico y su } \\
\text { correspondiente literatu- } \\
\text { ra docente. }\end{array}$ & $\begin{array}{l}\text { Materiales electrónicos e } \\
\text { impresos, así como ma- } \\
\text { terial complementario. }\end{array}$ & $\begin{array}{l}\text { Se manejará mediante } \\
\text { telecomunicación vía sa- } \\
\text { télite; se darán clases; } \\
\text { se transmitirán videos y } \\
\text { slides; y mediante el do- } \\
\text { cument camera se am- } \\
\text { pliará parte de algunos } \\
\text { documentos impresos. }\end{array}$ \\
\hline
\end{tabular}

30 Norma Barrios Fernández, "Los estudios a distancia de la carrera de información científicotécnica y bibliotecología en Cuba”, en: Encuentro de Educadores e Investigadores de Bibliotecología, Archivología y Ciencia de la Información de Iberoamérica y el Caribe (3 : 1996 : Recinto de Río Piedras, Puerto Rico), Recinto de Río Piedras, UPR: Escuela Graduada de Bibliotecología y Ciencia de la Información, 1996. pp.1-12. Documento de trabajo I mesas redondas Comisión Educación a Distancia núm.2ª

31 Noemí Conforti, "Universidad Nacional de Mar del Plata : la educación a distancia en la formación de los bibliotecarios”, en: Reunión Nacional de bibliotecarios (35 : 2001: Buenos Aires, Argentina), Las bibliotecas, el ciudadano y el derecho a la información. Buenos Aires, Asociación de Bibliotecarios Graduados de la República Argentina, 2001, [pág. varía]. 


\begin{tabular}{|l|l|l|l|}
\hline $\begin{array}{l}\text { Evaluación del aprendi- } \\
\text { zaje }\end{array}$ & $\begin{array}{l}\text { Se realiza mediante exa- } \\
\text { men final de cada asig- } \\
\text { natura. }\end{array}$ & $\begin{array}{l}\text { Para tener derecho a exa- } \\
\text { men final deben aprobar } \\
\text { el } 100 \% \text { de los trabajos } \\
\text { prácticos y actividades } \\
\text { integradoras. } \\
\text { Aprobar dos exámenes } \\
\text { parciales. }\end{array}$ & $\begin{array}{l}\text { Lo realiza personal dife- } \\
\text { rente a los que impartie- } \\
\text { ron la asignatura. }\end{array}$ \\
\hline $\begin{array}{l}\text { Requisitos para la titula- } \\
\text { ción }\end{array}$ & $\begin{array}{l}\text { Culminan con la realiza- } \\
\text { ción de un examen esta- } \\
\text { tal en el que se evalúa el } \\
\text { contenido del programa } \\
\text { del examen estatal. }\end{array}$ & $\begin{array}{l}\text { Una práctica profesional } \\
\text { y un examen de defensa } \\
\text { de lo que realizaron en } \\
\text { esa práctica. }\end{array}$ & $\begin{array}{l}\text { No secifica en la } \\
\text { fuente. }\end{array}$ \\
\hline
\end{tabular}

